\section{On some theorems of S. Saks}

by

\section{A. ALEXIEWICZ (Poznań).}

In a series of papers ([6]-[8]) SAKs has proved some theorems concerning the structure of the sequences of linear operations defined in Banach spaces, with values in the space $\boldsymbol{S}$ of measurable functions defined on an interval $I$. These theorems state that the interval $I$ can be split into two measurable sets on one of which the sequence has a particular structure (e. g. is bounded or convergent almost everywhere) and on the second of which it is deprived of this property except for a set of the first category of values of the argument.

I shall prove that all these theorems and a large class of analogous results may be considered as a particular case of a general theorem concerning the structure of one linear operation depending on a parameter. In this paper $I$ am dealing with the "individual" case, i. e. with the behaviour of the operation at individual values of the parameter, the treatment of the behaviour in mean being left to another paper.

1. We will be concerned with two $F$-spaces ([1], p. 35) $X_{\text {and }}$ $Y$, and an abstract set $T$ with a $\sigma$-ring $\mathbb{E}$ of subsets of $T$ (called measurable sets) on which a $\sigma$-additive measure $\mu$ is defined. Wo suppose that $T \in \mathbb{E}$ and $\mu(T)<\infty$. The measure $\mu$ defines a measure space $(T, \mathbb{E}, \mu)$ : introducing the distance of two sets $E_{1}, E_{2} \in \mathbb{E}$ by the formula

$$
\varrho\left(E_{1}, E_{2}\right)=\mu\left(E_{1}-E_{2}\right)+\mu\left(E_{2}-E_{1}\right)
$$

and considering two sets subject to the condition $\varrho\left(E_{1}, E_{2}\right)=0$ as one element of the space $(T, \mathfrak{E}, \mu)$ we get a complete metric space.

We suppose that the measure $\mu$ is separable, i. e. that the space $(T, \mathbb{E}, \mu)$ is so.

In the sequel $U(x, t)$ will denote an operation from $X \times T$ to $Y$ satisfying the following conditions:

(a) $U\left(x_{1}+x_{2}, t\right)=U\left(x_{1}, t\right)+U\left(x_{2}, t\right)$ a. e. $\left.{ }^{1}\right)$ in $T$,

(b) $x_{n} \rightarrow 0$ implies $\lim$ as $\left\|U\left(x_{n}, t\right)\right\|=0$,

(c) given any $x$ and any open set $S \subset Y$, the set $\underset{t}{E}\{U(x, t) \epsilon S\}$ is measurable.

These conditions imply

(d) $U(\lambda x, t)=\lambda U(x, t)$ a. e. in $T$.

Finally, we put for any set $R \subset Y$

$$
\Theta(x)=\underset{t}{\boldsymbol{t}}\{U(x, t) \epsilon R\}
$$

this set will be denbted also, if necessary, by $\Theta_{R}(x)$.

Lemma 1. For any set $R \subset Y$, measurable $(B)$, the set $\Theta(x)$ is measurable.

The easy proof is left to the reader.

Lemma 2. For any set $R \subset Y$, measurable $(B)$, and any $H \in \Subset$ the sets

$$
\begin{aligned}
& \boldsymbol{A}(H, R)=\underset{x}{\boldsymbol{E}}\{\mu(H-\Theta(x))<\varepsilon\}, \\
& \boldsymbol{B}(\boldsymbol{H}, R)=\underset{x}{\boldsymbol{E}}\{\mu(H-\Theta(x)) \leqslant \varepsilon\}
\end{aligned}
$$

are measurable $(\boldsymbol{B})$.

Proof. We prove first that, the set $R$ being, elosed, the set $B(H, R)$ is also closed. For, let $x_{n} \epsilon B(H, R)$, then

$$
\lim \operatorname{as}\left\|U\left(x_{n}, t\right)-U\left(x_{0}, t\right)\right\|=0 .
$$

Hence there exists a subsequence $\left\{x_{n_{i}}\right\}$ such that

$$
\left\|U\left(x_{n_{t}}, t\right)-U\left(x_{0}, t\right)\right\| \rightarrow 0
$$

except in a set $N_{\epsilon} \mathbb{E}$ of measure 0 . Put

$$
\Theta=\lim _{i} \sup \theta\left(x_{n_{i}}\right) \text {. }
$$

Then $\mu(H-\Theta)=\mu\left(\liminf \left(H-\Theta\left(x_{n_{i}}\right)\right)\right) \leqslant \varepsilon$. If $t_{\epsilon} \Theta-N$, then there

\footnotetext{
1) a. e. = almost everywhere.
} 
exists a sequence of indices $\left\{k_{i}\right\}$ (depending on $t$ ) such that $t \epsilon \Theta\left(x_{n_{k_{i}}}\right)$, hence

$$
\left\|U\left(x_{n_{k_{i}}}, t\right)-U\left(x_{0}, t\right)\right\| \rightarrow 0,
$$

and since $U\left(x_{n_{k_{i}}}, t\right) \in R$, the closedness of the set $R$ implies $U\left(x_{0}, t\right) \in R$. It follows $\Theta-N \subset \Theta\left(x_{0}\right)$; thus we have shown that the set $B(H, R)$ is closed.

The formula

$$
\underset{x}{E}\{\mu(H-\Theta(x))<\varepsilon\}=\sum_{n=1}^{\infty} \underset{x}{E}\left\{\mu(H-\Theta(x)) \leqslant\left(1-\frac{1}{n}\right) \varepsilon\right\}
$$

implies that for any closed set $R$ the set $A(H, R)$ is of $F_{\sigma}$ type.

To prove the lemma in general case it suffices to show that

$$
R_{1}, R_{2}, \ldots
$$

being any monotone sequence of sets, with the limit $R,(B)$-measurability of the sets $A\left(H, R_{n}\right)$ and $B\left(H, R_{n}\right)$ implies the same relatively to the sets $A(H, R)$ and $B(H, R)$.

Put

$$
\Omega_{n}(x)=\underset{t}{t}\left\{U(x, t) \in R_{n}\right\}
$$

and suppose first the sequence (3) to be increasing. It is obvious that

$$
\Theta_{R}(x)=\sum_{n=1}^{\infty} \Omega_{n}(x)=\Omega(x) .
$$

We will prove that

$$
\underset{x}{E}\{\mu(H-\Omega(x))<\varepsilon\}=\sum_{n=1}^{\infty} \underset{x}{E}\left\{\mu\left(H-\Omega_{n}(x)\right)<\varepsilon\right\} .
$$

For, if $x$ belongs to the set on the left hand side of the formula (4), then $\mu(H-\Omega(x))<\varepsilon$. The sequence of sets $\Omega_{1}(x), \Omega_{2}(x), \ldots$ being increasing,

$$
\begin{gathered}
H-\Omega(x)=\lim _{n}\left(H-\Omega_{n}(x)\right), \\
\mu(H-\Omega(x))=\lim _{n} \mu\left(H-\Omega_{n}(x)\right) ;
\end{gathered}
$$

hence there exists an index $n$ for which $\mu\left(H-\Omega_{n}(x)\right)<\varepsilon$, i. e. $x$ belongs to the set on the right hand side. Conversely, if $x$ belongs to the set on the right hand side, then there exists an index $n$ such that $\mu\left(H-\Omega_{n}(x)\right)<\varepsilon$, and since $H-\Omega(x) \subset H-\Omega_{n}(x)$, $x$ belongs also to the set on the left hand side.
The formula (4) shows that the set $A(H, R)$ is measurable $(B)$ if the sets $A\left(H, R_{n}\right)$ are so. $(B)$-measurability of the set $B(H, R)$ follows by the formula

$$
\underset{x}{E}\{\mu(H-\Theta(x)) \leqslant \varepsilon\}=\prod_{n=1}^{\infty} E\left\{\mu(H-\Theta(x))<\left(1+\frac{1}{n}\right) \varepsilon\right\} .
$$

Suppose now that the sequence (3) is decreasing. Then

$$
\Theta_{R}(x)=\prod_{n=1}^{\infty} \Omega_{n}(x)=\Omega^{*}(x)
$$

and $(B)$-measurability of the set $B(H, R)$ follows by the formula

$$
\underset{x}{E}\left\{\mu\left(H-\Omega^{*}(x)\right) \leqslant \varepsilon\right\}=\prod_{n=1}^{\infty} E\left\{\mu\left(H-\Omega_{n}(x)\right) \leqslant \varepsilon\right\},
$$

analogous to the formula (4); $(B)$-measurability of the set $A(H, R)$ follows by the formula (2).

Lemma 3. Let the set $R$ be linear and measurable $(B)$. If the set $W=E\{\mu(H-\Theta(x))<\varepsilon\}$ is of the second category, then the set $V=\underset{x}{E}\{\mu(H-\Theta(x))<2 \varepsilon\}$ is residual $\left.{ }^{2}\right)$.

Proof. Write $P \dot{C} Q$ if $\mu(Q-P)=0$. The formula

$$
\Theta(x) \Theta(y) \dot{C} \Theta(x-y)
$$

implies

$$
H-\Theta(x-y) \dot{C}[H-\Theta(x)]+[H-\Theta(y)],
$$

hence $\mu(H-\Theta(x-y))<2 \varepsilon$ if $x, y \in W$. The set $W$ being measurable $(B)$ by Lemma 2, if the elements $x, y$ run down through the set $W$ the elements $x-y$ fill a sphere $S(0, r)$ with centre 0 and radius $r$, except a set $N$ of the first category. Hence

$$
S(0, r)-N \subset \underset{x}{E}\{\mu(H-\Theta(x))<2 \varepsilon\} .
$$

The lemma results by the formula $\mu(\Theta(\lambda x)-\Theta(x))=0$.

Lemma 4. The set $R$ being linear and measurable $(B)$, the set $Z=E\{\mu(H-\Theta(x))=0\}$ is either of the first category or identical with $X$.

2) The set $W$ is said to be residual if its complement is of the first category. 
Proof. By Lemma 3 this set is residual, hence every translation of the set $Z$ has common points with $Z$. It suffices to note tion of the set $Z$ has colloments to $Z$.

that the difference of the set $R$ be linear and measurable $(B)$. Then

Theorem 1 . Let the set $R$ be linear and measurable and a residual set $Z \subset X$ such that

(i) $U(x, t) \in R$ for any $x$ a. e. in $A$,

(ii) $U(x, t) \operatorname{non} \epsilon R$ for any $x \in \mathbb{Z}$ a. e. in $B$.

Proof. Denote by $\mathfrak{A}$ the class of the sets $Q$ belonging to $\mathbb{E}$ and subject to the condition

$$
\mu(Q-\Theta(x))=0 \text { for every } x .
$$

Let $\sigma$ denote the supremum of the measures of the sets of $\mathfrak{A}$. There exist sets $Q_{n}$ in $\mathfrak{A}$ such that $\mu\left(Q_{n}\right) \rightarrow \sigma$ and $\mu\left(Q_{1}\right) \leqslant \mu\left(Q_{2}\right) \leqslant \ldots$ Put

$$
A=\sum_{n=1}^{\infty} Q_{n}, \quad B=T-A .
$$

Then (i) is evidently satisfied.

Suppose now (ii) not to be satisfied. Then the set

$$
\underset{x}{E}\{\mu(B \Theta(x))>0\}
$$

is of the second eategory, hence there exists an $\alpha>0$ such that the set $Q=E\{\mu(B \Theta(x))>a\}$ is of the second category. Let $\mathfrak{B}$ denote the class of the sets of $\mathbb{E}$ contained in $B$ of measure not less than $\alpha$; there exist sets $M_{n} \in \mathfrak{B}$ such that the sequence $M_{1}, M_{2}, \ldots$ is dense in $\mathfrak{B}$. Write

$$
X_{m n}=E x\left\{\mu\left(M_{n}-\Theta(x)\right)<\frac{1}{2^{m}}\right\} .
$$

Since $Q \subset \sum_{n=1}^{\infty} X_{m n}$, there exists an index $n_{m}$ such that the set

$$
\underset{x}{E}\left\{\mu\left(M_{n_{m}}-\Theta(x)\right)<\frac{1}{2^{m}}\right\}
$$

is of the second category, hence by Lemma 3 the sets

$$
W_{m}=\underset{x}{E}\left\{\mu\left(\boldsymbol{M}_{n_{m}}-\Theta(x)\right)<\frac{1}{2^{m-1}}\right\}
$$

are residual. Put

$$
W=\prod_{n=1}^{\infty} W_{m}, \quad M=\underset{m}{\lim \sup } M_{n_{m}} .
$$

The set $W$ is residual and $\mu(M) \geqslant \lim \sup \mu\left(M_{n_{m}}\right) \geqslant \alpha$. Let $x \epsilon W$, then

$$
\begin{gathered}
\mu(M-\Theta(x))=\mu\left(\prod_{s=1}^{\infty} \sum_{m=s}^{\infty}\left(M_{n_{m}}-\Theta(x)\right)\right) \\
\leqslant \mu\left(\sum_{m=s}^{\infty}\left(M_{n_{m}}-\Theta(x)\right)\right) \leqslant \sum_{m=s}^{\infty} \mu\left(M_{n_{m}}-\Theta(x)\right)<\frac{1}{2^{s-2}},
\end{gathered}
$$

hence $\mu(M-\Theta(x))=0$. By Lemma $4 \mu(M-\Theta(x))=0$ for every $x$ in $X$. This is however impossible since $\mu(A+M) \geqslant \sigma+\alpha$ and the set $A+M$ has the property (i).

Remark 1 . Theorem 1 is also valid under hypothesis of the measure $\mu$ being $\sigma$-finite on $T$ ([3], p. 31).

Remark 2. In order to apply Theorem 1 we must first show that the operation $U(x, t)$ satisfies the postulate (c). A sufficient condition for (c) is Bochner-measurability ([2], p. 264) of $U(x, t)$ considered for fixed $x$ as vector valued function from $T$ to $Y$. In the case of $Y$ being separable and of $B_{0}$ type (MAzur and ORLICZ [4]) the following criterion due to PETrIs ([5], p. 278) is useful: the function $y(t)$ from $T$ to $Y$ is Bochner-measurable if for every linear functional $\eta y$ belonging to a fundamental set $\Gamma$, the function $\eta y(t)$ is measurable. Here by a fundamental set is meant any set of linear functionals, which for every $y, \varepsilon>0$, and $k$ contains a functional $\eta$ such that $|y|_{k}-\varepsilon \leqslant|\eta y|<|y|_{k},|y|_{k}$ denoting the $k$-th pseudonorm in the space $Y$

Theorem 1 implies immediately the following

Corollary. Let $\left\{R_{n}\right\}$ be an increasing sequence of linear sets measurable $(B)$. Then there exists a decomposition $T=\sum_{n=0}^{\infty} A_{n}$ into measurable sets, and a residual set $Z$ such that

(i) $U(x, t) \epsilon R_{n}$ for every $x$ a.e. in $A_{n}(n=1,2, \ldots)$,

(ii) $U(x, t)$ non $\epsilon \sum_{n=1}^{\infty} R_{n}$ for every $x \in Z$ a.e. in $A_{0}$. 
2. Now we will supply some applications of Theorem 1. To obtain the theorems of Saks and their analoga, we choose as $Y$ the space $s$ of the sequences $y=\left\{\alpha_{n}\right\}$ with the norm

$$
\|y\|=\sum_{n=0}^{\infty} \frac{1}{2^{n}} \frac{\left|\alpha_{n}\right|}{1+\left|\alpha_{n}\right|} .
$$

This space is separable and of $B_{0}$-type.

Let $\boldsymbol{S}$ denote the space of the real valued functions $y=y(t)$ defined in $T$ and measurable $\mu$. An operation $V(x)=V(x, t)$ from $X$ to $S$ is said to be linear if $V\left(x_{1}+x_{2}, t\right)=V\left(x_{1}, t\right)+V\left(x_{2}, t\right)$ a. e. ancl $x_{n} \rightarrow x_{0}$ implies asymptotical convergence of $V\left(x_{n}, t\right)$ to $V\left(x_{0}, t\right)$. Any sequence $\left\{V_{n}(x, t)\right\}$ of linear operations from $X$ to $\boldsymbol{S}$ may be considered as an operation $U(x, t)$ from $X \times T$ to $s$, since for fixed $x, t$ it defines a sequence of reals, i. e. an element of $s$. It is easy to verify that the operation $U(x, t)$ satisfies the postulates (a)-(c). In particular (c) results by the mentioned criterion of Pettis.

Denote successively by $R_{1}, \ldots, R_{4}$ the classes of the sequences $y=\left\{a_{n}\right\}$ with the following properties:

$1^{\circ}$ the sequence $\left\{a_{n}\right\}$ is bounded,

$2^{\circ}$ the sequence $\left\{\alpha_{n}\right\}$ converges,

$3^{\circ}$ the sequence $\left\{\alpha_{n}\right\}$ converges to 0 ,

$$
4^{\circ} \quad \sum_{n=0}^{\infty}\left|\alpha_{n}\right|^{p}<\infty \quad(p>0) \text {. }
$$

These classes considered as subsets of the space $s$ are linear and measurable $(B)$. Theorem 1 applied to the operation $U(x, t)$ gives now

Theorem 2. Let $V_{n}(x, t)$ be a sequence of linear operations from $X$ to $\boldsymbol{S}$; then there exist decompositions $T=A_{1}+B_{1}=\ldots=A_{4}+B_{4}$ and residual sets $Z_{1}, \ldots, Z_{4}$ such that ${ }^{3}$ )

$\left(a_{1}\right) \varlimsup\left|V_{n}(x, t)\right|<\infty$ for every $x$ a.e. in $A_{1}$,$$
\lim _{n \rightarrow \infty}
$$

$\left(b_{1}\right) \varlimsup_{n \rightarrow \infty}\left|V_{n}(x, t)\right|=\infty$ for every $x \in Z_{1}$ a.e. in $B_{1}$,

$\left(a_{2}\right) \lim _{n \rightarrow \infty} V_{n}(x, t)$ exists for every $x$ a.e. in $A_{2}$,

3) The cases $\left(a_{1}\right)-\left(b_{2}\right)$ were proved by Saks [7]. $\left(b_{2}\right) \quad \lim V_{n}(x, t)$ does not exist for every $x \in Z_{2}$ a. e. in $B_{2}$ $n \rightarrow \infty$

$\left(a_{3}\right) \lim _{n \rightarrow \infty} V_{n}(x, t)=0$ for exery $x$ a.e. in $A_{3}$.

$\left(b_{3}\right) \varlimsup_{n \rightarrow \infty}\left|V_{n}(x, t)\right|>0$ for every $x \in Z_{3}$ a.e. in $B_{3}$,

$\left(a_{4}\right) \sum_{n=0}^{\infty}\left|V_{n}(x, t)\right|^{p}<\infty$ for every $x$ a. e. in $A_{4}$,

$\left(\mathrm{b}_{4}\right) \sum_{n=0}^{\infty}\left|V_{n}(x, t)\right|^{p}=\infty$ for every $x \in Z_{4}$ a. e. in $B_{4}$.

Denote now by $R_{\varrho}$ the set composed of these $y=\left\{\alpha_{n}\right\} \in \boldsymbol{s}$ for which the series $\sum_{n=0}^{\infty} \alpha_{n} \zeta^{n}$ has the radius of convergence not less than $\varrho$. This set is measurable $(B)$; this follows by formula

$$
R=\prod_{k=1}^{\infty} \sum_{n=1}^{\infty} \prod_{m=n}^{\infty} E_{k m}
$$

where

$$
E_{k m}=\underset{y}{E}\left\{y=\left\{\alpha_{n}\right\}, \quad\left|a_{m}\right|<\left(\frac{k}{k \varrho-\varrho}\right)^{m}\right\} .
$$

Applying the Corollary we get easily

Theorem 3. Under the hypotheses of Theorem 2 there exists for every $\varepsilon>0$ a decomposition $T=A+B+C$, and a residual set $Z$ such that

(a) the series $\sum_{n=0}^{\infty} V_{n}(x, t) \zeta^{n}$ converges for every $x$ and every $|\zeta|<\varrho$ a.e. in $A$

(b) the series $\sum_{n=0}^{\infty} V_{n}(x, t) \zeta^{n}$ diverges for every $x \in Z$ and every $|\zeta|>0$
in $B$, a.e. in $B$,

(c) $\mu(C)<\varepsilon$.

As a second application we give an analogous theorem dealing with operations depending on a continuous parameter. Let $C^{*}$ denote the space of the functions $y=y(\lambda)$ defined for $a \leqslant \lambda<b$ (where $-\infty<a<b \leqslant+\infty)$ and continuous there. The norm is defined as

$$
\|y\|=\sum_{n=0}^{\infty} \frac{1}{2^{n}} \frac{|y|_{n}}{1+|y|_{n}}
$$


where $|y|_{n}=\sup _{a \leqslant \lambda \leqslant b_{n}}|y(\lambda)|, \quad b_{n}=b-\frac{1}{n+1}$ if $b<+\infty$ and $b_{n}=n$ if $b=\infty$. This space is separable and of $B_{0}$-type.

Denote now successively by $R_{1}, \ldots, R_{6}$ the subsets of $C^{\star}$ composed of the functions $y=y(\lambda)$ for which respectively

$1^{\circ}|y(\lambda)|$ is bounded in $[a, b)$,

$2^{\circ} \lim y(\lambda)$ exists,$$
\lambda \rightarrow b
$$

$3^{\circ} \int_{a}^{b}|y(\lambda)|^{p} d \lambda<\infty \quad(p>0)$,

$4^{\circ} \int_{a}^{b-} y(\lambda) d \lambda=\lim _{\tau \rightarrow b-} \int_{a}^{\tau} y(\lambda) d \lambda$ exists,

$5^{\circ} y(\lambda)$ is of bounded variation in $[a, b)$, i. e.

$$
\operatorname{var}_{a \leqslant \lambda<b} y(\lambda)=\sup _{a<\tau<b} \operatorname{var}_{a \leqslant \lambda \leqslant \tau} y(\lambda)<\infty
$$

$6^{\circ} y(\lambda)$ is absolutely continuous in $[a, b)$.

These sets are measurable $(B)$. We prove this for some of them.

Ad $R_{3}$. The sets

$$
Q_{n m}=\underset{y}{E}\left\{\int_{a}^{b_{n}}|y(\lambda)|^{\alpha} d \lambda \leqslant m\right\}
$$

$b_{n}$ being a sequence with limit $b$, are closed in $C^{*}$ and

$$
R_{3}=\sum_{m=1}^{\infty} \prod_{n=1}^{\infty} Q_{n m}
$$

Ad $R_{5}$. The sets

$$
\left.Q^{\prime}{ }_{n m}=\underset{\nu}{E} \underset{a \leqslant \lambda \leqslant b_{n}}{\operatorname{var}} y(\lambda) \leqslant m\right\}
$$

are closed in $C^{*}$ and

$$
R_{5}=\sum_{m=1}^{\infty} \prod_{n=1}^{\infty} Q_{n m}
$$

Ad $R_{6}$. Denote by $V\left(y ; \eta, b^{\prime}\right)$ the supremum of the sums

$$
\sum_{i}\left|y\left(\beta_{i}\right)-y\left(\alpha_{i}\right)\right|
$$

where $\left(\alpha_{i}, \beta_{i}\right)$ is any system of disjoint intervals contained in $\left[a, b^{\prime}\right]$ and $\sum_{i}\left|\beta_{i}-a_{i}\right|<\eta$, then put

$$
Q_{n m p}=\underset{y}{E}\left\{V\left(y ; \frac{1}{m}, b_{n}\right) \leqslant \frac{1}{p}\right\} .
$$

The sets $Q_{n m p}$ are closed and

$$
R_{6}=\prod_{p=1}^{\infty} \sum_{m=1}^{\infty} \prod_{n=1}^{\infty} Q_{n m p}
$$

Theorem 4. Let $W_{\lambda}(x, t)$ denote an operation which, for fixed $\lambda$, is linear from $X$ to $\boldsymbol{S}$ and for fixed $x, t$ depends continuously on the parameter $\lambda$ varying in $[a, b)$. Then there exist decompositions $T=A_{1}+B_{1}=\ldots=A_{6}+B_{6}$ and residual sets $Z_{1}, \ldots, Z_{6}$ such that $\left.{ }^{4}\right)$

$\left(a_{1}\right) \varlimsup_{\lambda \rightarrow b-}\left|W_{\lambda}(x, t)\right|<\infty$ for every $x$ a. e. in $A_{1}$,

$\left(b_{1}\right) \varlimsup_{\lambda \rightarrow b-}\left|W_{\lambda}(x, t)\right|=\infty$ for every $x \in Z_{1}$ a.e. in $B_{1}$,

$\left(a_{2}\right) \lim W_{\lambda}(x, t)$ exists for every $x$ a.e. in $A_{2}$, $x_{\lambda \rightarrow b-}$

$\left(b_{2}\right) \quad \lim W_{\lambda}(x, t)$ does not exist for every $x \in Z_{2}$ a.e. in $B_{2}$,

$\left(\mathrm{a}_{3}\right) \int_{a}^{b}\left|W_{\lambda}(x, t)\right|^{p} d \lambda<\infty$ for every $x$ a.e. in $A_{3}$,

$\left(\mathrm{b}_{3}\right) \int_{a}^{b}\left|W_{\lambda}(x, t)\right|^{p} d \lambda=\infty$ for every $x \in Z_{3}$ a.e. in $B_{3}$, a

$\left(a_{4}\right) \int_{a}^{b-} W_{\lambda}(x, t) d \lambda$ exists for every $x$ a.e. in $A_{4}$,

$\left(\mathrm{b}_{4}\right) \int_{a}^{b-} W_{\lambda}(x, t) d \lambda$ does not exist for every $x \in Z_{4}$ a.e. in $B_{4}$,

$\left(a_{5}\right) \operatorname{var} W_{\lambda}(x, t)<\infty$ for every $x$ a. e. in $A_{5}$, $a \leqslant \lambda<b$

$\left(\mathrm{b}_{5}\right) \underset{a \leqslant \lambda<b}{\operatorname{var}} W_{\lambda}(x, t)=\infty$ for every $x \in Z_{5}$ a.e. in $B_{5}$,

4) The cases $\left(a_{3}\right) \cdot\left(b_{4}\right)$ were proved by Orlicz (to be published in Studia Mathematica). 
$\left(a_{8}\right) W_{\lambda}(x, t)$ is $A C$ in $\lambda$ for every $x$ a. e. in $A_{6}$,

$\left(\mathrm{b}_{6}\right) W_{\lambda}(x, t)$ is not $A C$ in $\lambda$ for every $x \in Z_{6}$ a.e. in $B_{6}$.

Proof. Consider $W_{\lambda}(x, t)$ as operation $U(x, t)$ from $X \times T$ to $C^{*}$. It is easy to prove that the conditions (a)-(c) are satisfied. Now we apply Theorem 1.

Theorem 4 enables us to get results concerning the structures of some functional spaces.

Theorem 5. Let $X$ be an F-space composed of the functions $x=x(\zeta)$ of the complex variable $\zeta$, continuous for $|\zeta|<1$. Suppose that $\left\|x_{n}\right\| \rightarrow 0$ implies for $t=$ const $\operatorname{limas}_{n \rightarrow \infty} x_{n}\left(\lambda e^{i l}\right)=0$, and that addition. of elements and multiplication by reals are defined in usual sense. Under the above hypotheses there exist decompositions $[0,2 \pi]=$ $=A_{1}+B_{1}=\ldots=A_{3}+B_{3}$, and residual sets $Z_{1}, Z_{2}, Z_{3}$ such that

$\left(a_{1}\right) \varlimsup_{\lambda \rightarrow 1-}\left|x\left(\lambda e^{i t}\right)\right|<\infty$ for every $x$ a. e. in $A_{1}$,

$\left(b_{1}\right) \varlimsup_{\lambda \rightarrow 1-}\left|x\left(\lambda e^{i t}\right)\right|=\infty$ for every $x \in Z_{1}$ a.e. in $B_{1}$,

$\left(a_{2}\right) \lim _{\lambda \rightarrow 1} x\left(\lambda e^{i t}\right)$ exists for every $x$ a. e. in $A_{2}$, $\lim _{\lambda \rightarrow 1-}$

$\left(\mathrm{b}_{2}\right) \lim _{\lambda \rightarrow 1} x\left(\lambda e^{i t}\right)$ does not exist for every $x \in Z_{2}$ a.e. in $B_{2}$,

$\left(\mathrm{a}_{3}\right) \int_{0}^{1}\left|x\left(\lambda e^{i t}\right)\right|^{p} d \lambda<\infty$ for every $x$ a.e. in $A_{3}$,

$\left(\mathrm{b}_{3}\right) \int_{0}^{1}\left|x\left(\lambda e^{i t}\right)\right|^{p} d \lambda=\infty$ for every $x \in Z_{3}$ a.e. in $B_{3}$,

moreover if the space has the property that $x(\zeta)$ being an element of it the function $x\left(e^{i p} \zeta\right)$ belongs also to this space, then in every couple $A_{i}, B_{i}$ one of the sets is empty.

Proof. Put $U_{\lambda}(x, t)=x\left(\lambda e^{i t}\right)$ and apply Theorem 4. The second part is obvious.

\section{Bibliography.}

[1] S. Banach, Théorie des opérations linéaires, Monografie Matematyczne, Warszawa 1932.

[2] S. Bochner, Integration von Funktionen, deren Werte die Elemente eines Veltorraumes sind, Fundamenta Mathematicae 20 (1933), p. 262-276.
[3] P. R. Halmos, Measure Theory, New York 1950.

[4] S. Mazur and W. Orlicz, Sur les espaces métriques linéaires, Studia Mathematica 11 (1948), p. 184-208.

[5] B. J. Pettis, Integration in vector spaces, Transactions of the American Math. Soc. 44 (1938), p. 277-304.

[6] S. Saks, Sur les fonctionnelles de M. Banach et leur applications aux développements de fonctions, Fundamenta Mathematicae 10 (1928), p. 186-196.

[7] - On some functionals, Transactions of the American Math. Soc. 35 (1932), p. $549-556$.

[8] - On some functionals II, ibidem 41 (1937), p. 160-170.

(Reçu par la Rédaction le 2. \%.1951) 